\title{
Relación entre conductas sexuales de riesgo y funcionamiento familiar en estudiantes de 14 a 19 años de edad de instituciones públicas y privadas de Lima
}

\author{
Relationship between risky sexual behaviors and family functioning in students aged \\ 14 to 19 from public and private institutions in Lima
}

Analy Quiroz Muñoz', Jessy Estelo Quispe²

\begin{abstract}
RESUMEN
Objetivo: determinar la relación entre conductas sexuales de riesgo y funcionamiento familiar en estudiantes de 14 a 19 años de edad de instituciones públicas y privadas de Lima. Metodología: enfoque cuantitativo, diseño no experimental, tipo correlacional y de corte transversal ya que no se manipulan las variables. En la recolección de datos se aplicó el Cuestionario de Conducta Sexual y el Test de Percepción del Funcionamiento familiar (FF-SIL) (diseñado por Pérez, Cuesta, Louro y Bayarre en 1997). La población estuvo conformada por 201 estudiantes de 3ero, 4to y 5to del nivel secundario. Resultados: en la primera variable, los porcentajes significativos en la dinámica familiar son $34.3 \%$ y $36.8 \%$ en "Moderadamente funcional" y "Funcional" respectivamente, percibiéndose mayormente un entorno familiar "Adecuado"; asimismo, en la segunda variable, el $28.4 \%$ indican ser sexualmente activos, siendo la edad de 12 años la más precoz en la iniciación sexual. Por último, en la asociación entre ambas variables se obtiene correlación significativa entre Funcionamiento familiar y Relaciones sexuales en grupo $(p<0.05, p=.004)$, empero, se percibe relación negativa entre las demás conductas sexuales de riesgo y el funcionamiento familiar. Conclusión: existe relación negativa entre conductas sexuales de riesgo y funcionamiento familiar, sin embargo, se encontró relación positiva entre la conducta sexual "relaciones sexuales en grupo" y funcionamiento familiar.
\end{abstract}

Palabras clave: adolescencia, funcionamiento familiar, conductas sexuales de riesgo.

\begin{abstract}
Objective: to determine the relationship between risky sexual behaviors and family functioning in students aged 14 to 19 from public and private institutions in Lima. Methodology: quantitative approach, non-experimental design, correlational and cross-sectional type since the variables are not manipulated. In data collection, the Sexual Behavior Questionnaire and the Family Functioning Perception Test (FFSIL) (designed by Pérez, Cuesta, Louro and Bayarre in 1997) were applied. The population was made up of 201 students from the 3rd, 4th and 5th grades of the secondary level. Results: In the first variable, the significant percentages in family dynamics are $34.3 \%$ and $36.8 \%$ in "Moderately functional" and "Functional", respectively, with a "Adequate" family environment mostly perceived; likewise, in the second variable, $28.4 \%$ indicate that they are sexually active, being the age of 12 years the earliest in sexual initiation. Finally, in the association between both variables, a significant correlation is obtained between Family functioning and Group sexual relations $(p<0.05, p=.004)$, however, a negative relationship is perceived between the other risky sexual behaviors and family functioning. Conclusion: There is a negative relationship between risky sexual behaviors and family functioning, however, a positive relationship was found between sexual behavior "group sex" and family functioning.
\end{abstract}

Keywords: adolescence, family functioning, risky sexual behaviors.

${ }^{1}$ Universidad Peruana Unión, Lima, Perú.

Orcid ID: 0000-0002-8371-6252

${ }^{2}$ Universidad Peruana Unión, Lima, Perú.

Orcid ID: 0000-0002-0983-7937 


\section{INTRODUCCIÓN}

Aproximadamente, 16 millones de adolescentes entre los 15 y 19 años pasan por el proceso de alumbramiento cada año, los bebes nacidos de madres adolescentes constituyen el $11 \%$ de la totalidad de nacimiento en el mundo y el $95 \%$ ocurren en países en desarrollo (OMS, 2012), e indudablemente serán madres que asumirán roles de personas adultas a temprana edad siendo vulnerables a presentar alteraciones emocionales que afecten la consolidación de su autoestima y personalidad, y porque no el crecimiento y desarrollo del recién nacido.

La OMS estima unos 376 millones de personas que contraen anualmente alguna infección por trasmisión sexual (ITS): clamidiosis, gonorrea, sífilis o tricomoniasis; más de 500 millones de personas son portadoras del virus del herpes simple; más de 290 millones de mujeres están infectadas por el virus del papiloma humano; y en 2016, más de 988 mil mujeres embarazadas fueron diagnosticadas con sífilis, que tuvo por consecuencia la muerte de más de 200 mil recién nacidos (Korenromp et al., 2019; Looker et al., 2015; OMS, 2016; OMS, 2018; Sanjosé et al., 2007).

Según el Instituto Nacional de Estadísitica e Informática (INEI, 2015) en Perú el embarazo adolescente se presenta en un $14,6 \%$ de la población, los departamentos con las tasas más altas son Loreto, Ayacucho y Lima con 10,6\%, $22,3 \%$ y $25 \%$, respectivamente. Asimismo, entre los adolescentes la prevalencia del virus de inmunodeficiencia humana (VIH) llegó a 287 casos confirmados y notificados desde 1983 hasta mediados del 2017 (MINSA, 2017). Ambas situaciones afectan el desarrollo y proyecto de vida de los adolescentes e impactan en su entorno social y familiar.

En Cuba, se realizó un estudio analítico con el fin de identificar las conductas de riesgo asociadas a las ITS en 336 adolescentes de 14 y 19 años en 2015, 66 adolescentes formaron el grupo control y 132 el grupo estudio; los resultados indican que del mayor número de adolescentes entre 18 y 19 años, el $50.5 \%$ presentan cambios frecuentes de parejas sexuales, el $48 \%$ practican relaciones sexuales sin protección, y la percepción de riesgos de adquirir una ITS, ya sea en el grupo control o de estudio, fue de $23.2 \%$ y $40.3 \%$ respectivamente; concluyendo en que la falta de protección en las relaciones sexuales y las múltiples parejas sexuales se asocian significativamente al riesgo de contraer cualquier tipo de ITS (Vidal \& Hernández, 2017).

En Latinoamérica, un estudio realizado en Brasil con el objetivo de determinar la asociación entre el número de parejas sexuales y el consumo de alcohol en una muestra total de 1257 estudiantes entre 12 y 24 años, finaliza en que existe variedad de factores que predisponen a las conductas sexuales de riesgo en adolescentes, mostrando que dichas conductas riesgosas podrían tener un origen multifactorial, donde la influencia del grupo y la cultura muestra que el consumo excesivo de alcohol y su edad de inicio constituyen los principales factores de riesgo para el comportamiento sexual vulnerable, además de estar asociado a la cantidad de parejas sexuales (Mola et al., 2017).

Lima, Neves y Lima (2012) analizaron las asociaciones de dicha problemática entre distintas características sociodemográficas, tales como factores predictores: determinantes sociales, los estilos de vida durante la adolescencia, el sexo, el grupo de edad, el estatus socioeconómico, la etnia; y factores protectores: el entorno familiar, elementos personales y psicológicos.

Un adecuado funcionamiento familiar permitirá al adolescente desarrollar la capacidad para enfrentar y superar las dificultades familiares en cada una de las etapas del ciclo vital (Castellón \& Ledesma, 2012); por lo tanto, los adolescentes que crezcan en familias sin una adecuada interacción entre sus miembros, con carencias de afectividad y practica de inadecuados estilos de crianza, entre otros factores, serán más propensos a incursionar en conductas sexuales de riesgo (Andrade et al., 2006).

En Ecuador un estudio buscó determinar la influencia del funcionamiento familiar y la conducta sexual de riesgo en 71 estudiantes entre 14 y 19 años entre los años 2015 y 2016; los resultados indicaron que el $49.3 \%$ de la población habían iniciado su vida sexual, las conductas sexuales de riesgo identificadas fueron: inicio precoz de la vida sexual, no uso de preservativo, y múltiples parejas sexuales $(P<0,05)$ según sexo, asimismo, la mayoría mencionó conocer el preservativo como método de protección, sin embargo menos del $40 \%$ refirieron haber usado dicho método, el $54 \%$ de adolescentes que iniciaron su vida 
sexual pertenecían a familias disfuncionales y moderadamente funcionales con una tipología familiar ampliada y extensa (Pérez, Apupalo, \& Creagh, 2018).

En Huancavelica, Albornoz, Encalada, y Meza (2018) determinaron la relación entre el funcionamiento familiar percibido y las conductas de riesgo en 69 adolescentes de 15 y 19 años, los resultados muestran que del total de la población de estudio, el $32 \%$ corresponden a una familia funcional mientras que el $68 \%$ pertenecen a una familia disfuncional, el $75 \%$ presentó las conductas de mayor riesgo como son: consumo de alcohol, tabaco u otras drogas e inicio de vida sexual precoz; en suma, este estudio corrobora los datos presentados por las demás investigaciones con respecto a que, existe correlación entre funcionamiento familiar ineficaz y conductas de riesgo $(p=.000)$.

De igual forma, otro estudio con el mismo objetivo de asociación entre Funcionalidad familiar y Conducta sexual de riesgo en 69 estudiantes de 16 a 24 años de una Universidad Pública de Lima, los resultados evidencian que el $58 \%$ tienen adecuado funcionamiento familiar, el $23.2 \%$ tiene muy buen funcionamiento familiar y el $18.8 \%$ tiene regular funcionamiento familiar; y con respecto a las conductas sexuales, el $63.8 \%$ se encuentran fuera de riesgo sexual, el $21.7 \%$ están en bajo nivel de riesgo sexual, el $13 \%$ presentan conductas de riesgo, y el $1.4 \%$ practican conductas sexuales de alto riesgo, esto datos estadísticos muestran que a mayor funcionamiento familiar menos probable son la práctica de conductas sexuales de riesgo en los jóvenes (Mejía, 2019).

Los estudios detallados muestran la realidad de la población adolescente a nivel mundial y nacional, por ello mediante el presente estudio se pretende hallar la asociación entre conductas sexuales de riesgo y funcionamiento familiar en adolescentes peruanos de 14 a 19 años, los resultados permitirán la creación de programas de intervención y prevención, así como propuestas terapéuticas, además del respaldo de las investigaciones ya existentes y la creación de nuevas ideas de investigación.

Finalmente, como objetivo general de investigación se pretende establecer si existe relación significativa entre conductas sexuales de riesgo y funcionamiento familiar en estudiantes de 14 a 19 años de edad en instituciones públicas y privadas de Lima; asimismo, como objetivos específicos se pretende identificar, primero las conductas sexuales de riesgo que practican los estudiantes de media y segundo el nivel de funcionamiento familiar presente en los adolescentes encuestados.

\section{Conducta sexual de riesgo - CSR}

Son conductas sexuales repetidas y fuera de límite que exponen al individuo a situaciones donde su salud y la de otra persona se ven afectadas, comprometiendo su desarrollo psicosocial normal durante la infancia o adolescencia, principalmente por el contagio de ETS/SIDA y la probabilidad de contraer embarazos no deseados (Aliaga, 2017; Espada et al., 2003). Tal comportamiento sexual implica como riesgo a las relaciones sexuales con o sin uso de medidas de protección, múltiples parejas sexuales, ETS o Sida, consumo de drogas, alcohol y tabaco, embarazo precoz, abuso sexual y aborto (González, Molina, Montero, Martínez \& Leyton, 2007). Además, el significado que se le puede atribuir a los elevados índices de riesgo es por no usar el preservativo y el inicio precoz de las relaciones sexuales, fundamentado en la cognición inmadura de cada adolescente (Fierros, Rivera, \& Piña, 2011; Losa \& Gonzáles, 2018; Vidal \& Hernández, 2017).

\section{Funcionamiento familiar}

Es el resultado de los esfuerzos de los integrantes para afrontar los diferentes cambios que se producen en el entorno familiar, ya sea por la etapa del ciclo vital en el que se encuentre o por determinadas situaciones que causan tensión, no obstante, se logra un nivel de equilibrio, armonía y coherencia ante esos eventos (Casasa, Ramírez, \& Guillén, 2016).

Minuchin, alega que la funcionalidad o disfuncionalidad de la familia no se ajusta a la ausencia de problemas dentro de ésta sino por el contrario, el cómo afronte las dificultades y de la manera cómo se adecua a las circunstancias cambiantes de modo que mantiene una continuidad y fomenta el crecimiento de cada miembro que conforma el ambiente familiar (citado por Llaza \& Ocoruro, 2018).

\section{Adolescencia}

La OMS (2005) define la adolescencia como el período de vida en el cual el individuo adquiere 
madurez reproductiva, transita los patrones psicológicos de la niñez a la adultez y están en busca de su independencia (Águila, Díaz \& Díaz, 2018). Asimismo, está dividía en tres sub etapas denominadas adolescencia temprana, media y tardía (Breinbauer y Maddaleno, 2005). Cada sub etapa comprende los siguientes rangos de edad según Gutgesell y Payne (2004): adolescencia temprana, etapa que abarca las edades de 10 hasta los 13 o 14 años; adolescencia media, etapa que puede iniciar desde los 14 o 15 años hasta la edad de 16 a 17 años; y finalmente adolescencia tardía, que comprende a partir de los 17 a 19 años en adelante teniendo en cuenta el desarrollo y madurez psicosocial para dar paso a la adultez (Restrepo, 2010).

\section{METODOLOGÍA}

La presente investigación corresponde a un estudio no experimental y de corte transversal debido a que no se manipula las variables, asimismo se pretende recolectar los datos en un solo momento. El alcance del estudio es correlacional puesto que se busca identificar si existe relación significativa entre las variables funcionamiento familiar y conductas sexuales de riesgo (Hernández, Fernández \& Baptista, 2014).

\section{Participantes}

La población estuvo compuesta por estudiantes de Instituciones Educativas de Lima, el tipo de muestreo fue no probabilístico por conveniencia (López, 2004), obteniendo una muestra de 201 adolescentes de ambos sexos del $3^{\circ}$ al $5^{\circ}$ año de secundaria. Las edades de los encuestados oscilaban entre 14 a 19 años de ambos sexos, siendo el $68.2 \%$ adolescentes en etapa media y el $31.8 \%$ adolescentes en etapa tardía.

Por otro lado, respecto a las personas con las que vive la población, el $65 \%$ viven con ambos padres, y sólo el $16 \%$ viven con la madre.

\section{Instrumentos}

En la aplicación de los instrumentos se incluyó una declaración de consentimiento informado, también se elaboró preguntas sobre los datos sociodemográficos. Las variables fueron medidas a través del Cuestionario de Conducta Sexual de Riesgo y el Test de Percepción del Funcionamiento Familiar (FF-SIL).

\section{Cuestionario de Conducta Sexual de Riesgo}

Diseñado por Apaza, Quiroz y Estelo (2017) en base al trabajo de investigación desarrollado por Tarazona (2005) en Lima, Perú, estudio de tipo correlacional y diseño no experimental, descriptivo simple al consistir en el cálculo de coeficientes de validez y confiabilidad, con una población de 400 estudiantes entre hombres y mujeres de instituciones educativas nacionales y privadas procedentes de Pachacamac, Ate, Santa Anita, El Agustino y Cieneguilla; tal estudio aborda la asociación entre el comportamiento sexual y las variables psicológicas: satisfacción con la vida, autoestima, estereotipia genérica y locus de control, y por otro lado, con seis variables demográficas: edad, tipo de colegio, sexo, pareja, ocupación actual y habitabilidad, en estudiantes de 5to año del nivel secundario.

La Escala diseñada originalmente por Tarazona (2005) es un autorreporte de prácticas sexuales que consta de 12 ítems y 3 dimensiones (complejidad, antigüedad y periodicidad). La validez de constructo se realizó mediante la prueba $\mathrm{t}$ de Student mostrando alto nivel de validez (52.87) y en cuanto al Coeficiente Alpha de Cronbach se percibe una adecuada confiabilidad (0.66).

El proceso de validación del cuestionario elaborado por Apaza, Quiroz y Estelo (2017) se realizó mediante la estimación del Coeficiente $\mathrm{V}$ de Aikeen a partir de la opinión de un grupo de expertos y jueces para aceptar o rechazar ítems, procedimiento que consiste en hacer que un grupo de jueces expertos verifiquen la representatividad de los indicadores de la conducta que se mide y de los ítems propuestos (Alarcón, 1998); se calculó el Coeficiente de Validez $\mathrm{V}$ de Aiken, aceptándose los ítems que alcancen valores estadísticamente significativos al 0.05 en todas las áreas evaluadas (pertinencia, relevancia y claridad). El cuestionario representa adecuadamente el área del comportamiento sexual que pretende medir y además presenta vocabulario adecuado y claridad en su redacción. Posteriormente a su elaboración se aplicó en 300 estudiantes del nivel secundario correspondiente a la investigación científica "Estilos de afrontamiento y conducta sexual de riesgo en adolescentes de tres instituciones educativas del distrito de Lurigancho" realizada por Apaza, Quiroz y Estelo (2017). En tanto, el presente cuestionario evalúa las manifestaciones del comportamiento sexual 
a partir del autorreporte de prácticas sexuales constituida por 15 ítems de evaluación.

\section{Test de Percepción del Funcionamiento Familiar} (FF-SIL)

Diseñado por Isabel Louro el cual permite diagnosticar el funcionamiento familiar, es decir, identificar la dinámica relacional que se da entre los miembros de una familia constituido por 7 dimensiones (cohesión, armonía, comunicación, adaptabilidad, afectividad, rol, y permeabilidad). La validez del contenido se realizó mediante la participación de 25 expertos, quienes demostraron la pertenencia de las características del inventario, así mismo establecieron el valor de cada una de ellas en la afectación de la salud familiar. Los criterios para validar la prueba fueron los criterios Murayama, en el cual se evaluó que los ítems sean razonables y comprensibles, se obtuvo que cada enunciado correspondía a la categoría designada, asimismo se evaluó que los componentes estén claramente definidos; por lo tanto, los jueces determinaron que los ítems cumplían con los criterios establecidos.

La consistencia de la prueba tiene coeficiente global de 0.84 demostrando homogeneidad entre los reactivos; en la confiabilidad temporal se obtuvo un valor de 0.93 , demostrando que los resultados de la prueba se mantienen durante el tiempo (Louro, 2003).

En la aplicación del Test de Funcionalidad Familiar de Isabel Louro, se tomó en cuenta el estudio realizado por Panuera (2018), investigación de tipo descriptivo correlacional titulada "Funcionalidad familiar, estilos parentales y sintomatología depresiva en adolescentes de 3ero, 4to y 5to grado de secundaria de una institución educativa estatal en Chorrillos, Lima" en 412 adolescentes. La validez de contenido para tal adaptación psicométrica se realizó mediante el método de criterio de jueces, se contó con la participación de 5 expertos, los resultados indicaron que la prueba es válida, observándose un $\mathrm{V}$ de Aiken de 1.00, concluyéndose que todos los ítems deben permanecer en la escala.

El análisis fue estudiado a partir del coeficiente de correlación ítem-test corregida, se encontró que existía una correlación directa entre los ítems y la prueba. Así también, se observó una adecuada confiabilidad, siendo el alfa de Cronbach igual a .88, lo que permite aseverar que con esta prueba se obtienen puntajes consistentes.

En cuanto a la estructura, el tipo de respuesta es Likert, compuesto por 14 ítems, expone una serie de situaciones que se pueden suscitar o no en la familia, para cada situación existe una escala de 5 respuestas cualitativas: Casi nunca, Pocas veces, A veces, Muchas veces, y Casi siempre; la puntuación total es la suma de los mismos, para finalmente asignarle un diagnóstico de funcionamiento familiar (Louro, 2004).

\section{Análisis de datos}

A fin de contrastar la hipótesis planteada y estudiar los datos se utilizó el programa estadístico SPSS 26.0, haciendo uso de las Tablas de Frecuencia y Tablas Cruzadas, así como el estadístico chi-cuadrado de Pearson para analizar los resultados de los datos y cumplir con el objetivo general.

\section{RESULTADOS}

\section{Funcionamiento familiar}

En la tabla $\mathrm{N}^{\circ} 1$ se aprecia que el $36.8 \%$ de adolescentes muestran una dinámica familiar moderadamente funcional, el $34.3 \%$ se hallan en la dimensión familiar de tipo funcional, sin embargo, el $23.4 \%$ presentan una familia disfuncional, y únicamente el $5.5 \%$ vive en un entorno familiar severamente disfuncional.

Tabla 1

Análisis descriptivo del funcionamiento familiar en adolescentes de 14 a 19 años

\begin{tabular}{lll} 
& $\mathrm{n}$ & $\%$ \\
Severamente disfuncional & 11 & $5.5 \%$ \\
Disfuncional & 47 & $23.4 \%$ \\
Moderadamente funcional & 74 & $36.8 \%$ \\
Funcional & 69 & $34.3 \%$ \\
\hline
\end{tabular}

En la tabla $\mathrm{N}^{\circ} 2$ se observa que, en su mayoría, el sexo masculino $(39.3 \%)$ demuestra una dinámica familiar de tipo funcional, en tanto que el sexo femenino (41.9\%) presenta una familia moderadamente funcional; asimismo, el $29.8 \%$ de varones se encuentra en la dimensión de 
familia moderadamente funcional, mientras que el $30.8 \%$ de mujeres corresponden a una familia funcional; sin embargo, el $27.4 \%$ de hombres y el $20.5 \%$ de mujeres presentan el tipo de familia disfuncional; y sólo el $3.6 \%$ de varones y el $6.8 \%$ de mujeres se hallan en la dimensión de familia severamente disfuncional.

Tabla 2

Análisis descriptivo del funcionamiento familiar según sexo

\begin{tabular}{|c|c|c|c|c|}
\hline & \multicolumn{4}{|c|}{ Sexo } \\
\hline & \multicolumn{2}{|c|}{ Masculino } & \multicolumn{2}{|c|}{ Femenino } \\
\hline & $\bar{n}$ & $\%$ & $n$ & $\%$ \\
\hline Severamente disfuncional & 3 & $3.6 \%$ & 8 & $6.8 \%$ \\
\hline Disfuncional & 23 & $27.4 \%$ & 24 & $20.5 \%$ \\
\hline Moderadamente funcional & 25 & $29.8 \%$ & 49 & $41.9 \%$ \\
\hline Funcional & 33 & $39.3 \%$ & 36 & $30.8 \%$ \\
\hline
\end{tabular}

La tabla $\mathrm{N}^{\circ} 3$ se divide en dos secciones, la primera parte corresponde a la adolescencia media (14 a 16 años), de los cuales el $24.4 \%$ pertenece a una familia funcional, el $23.4 \%$ a una familia moderadamente funcional, el $16.9 \%$ a una familia disfuncional, y sólo el $3.5 \%$ a una familia severamente disfuncional; la segunda parte corresponde a la adolescencia tardía (17 a 19 años), donde el $13.4 \%$ pertenece a una familia moderadamente funcional, el $10 \%$ a una familia funcional, el $6.5 \%$ a una familia disfuncional, y solo el $2 \%$ a una familia severamente disfuncional.

Tabla 3

Análisis descriptivo del funcionamiento familiar según edad

\begin{tabular}{|c|c|c|c|c|}
\hline & \multicolumn{4}{|c|}{ Edad } \\
\hline & \multicolumn{2}{|c|}{ Adolescencia media } & \multicolumn{2}{|c|}{ Adolescencia tardía } \\
\hline & \multicolumn{2}{|c|}{14 a 16} & \multicolumn{2}{|c|}{17 a 19} \\
\hline & $\mathbf{n}$ & $\%$ & $\mathrm{n}$ & $\%$ \\
\hline Severamente disfuncional & 7 & $3.5 \%$ & 4 & $2 \%$ \\
\hline Disfuncional & 34 & $16.9 \%$ & 13 & $6.5 \%$ \\
\hline Moderadamente funcional & 47 & $23.4 \%$ & 27 & $13.4 \%$ \\
\hline Funcional & 49 & $24.4 \%$ & 20 & $10 \%$ \\
\hline
\end{tabular}

\section{Conducta sexual de riesgo - Variable 2}

En la figura $\mathrm{N}^{\circ} 1$ se estima que el $28.4 \%$ de los adolescentes encuestados refieren haber iniciado su vida sexual, mientras que el $71.6 \%$ restante menciona no haber iniciado su vida sexual. 
Figura 1

Inicio temprano de vida sexual

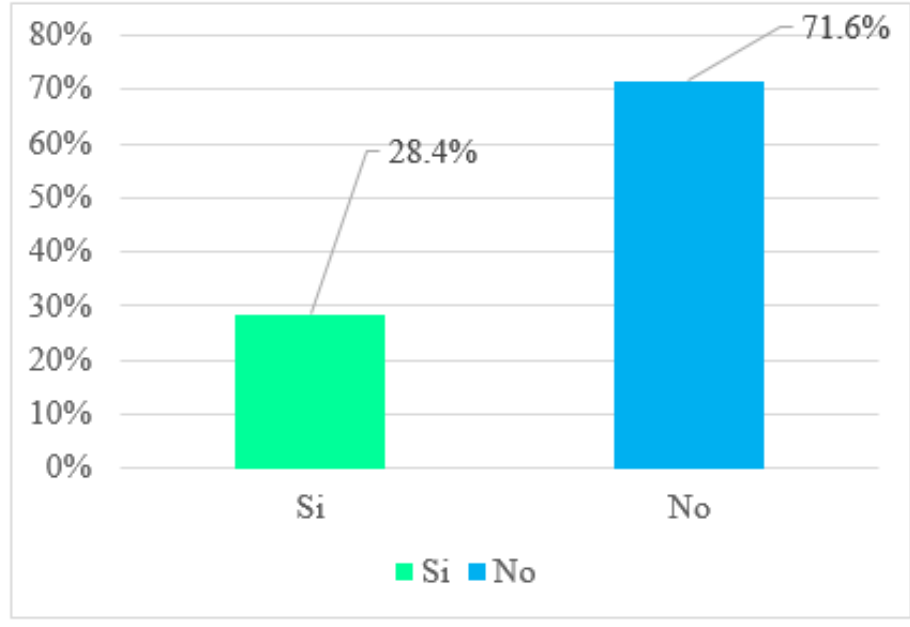

En la figura $\mathrm{N}^{\circ} 2$ se considera que el $22.8 \%$ inició a los 15 años, el $21.1 \%$ a los 17 años, el $15.8 \%$ a los 14 años, el $14 \%$ a los 16 años, el $12.3 \%$ a los 13 años, el $8.8 \%$ a los 18 años, el $3.5 \%$ a los 19 años y finalmente, el $1.8 \%$ a los 12 años.

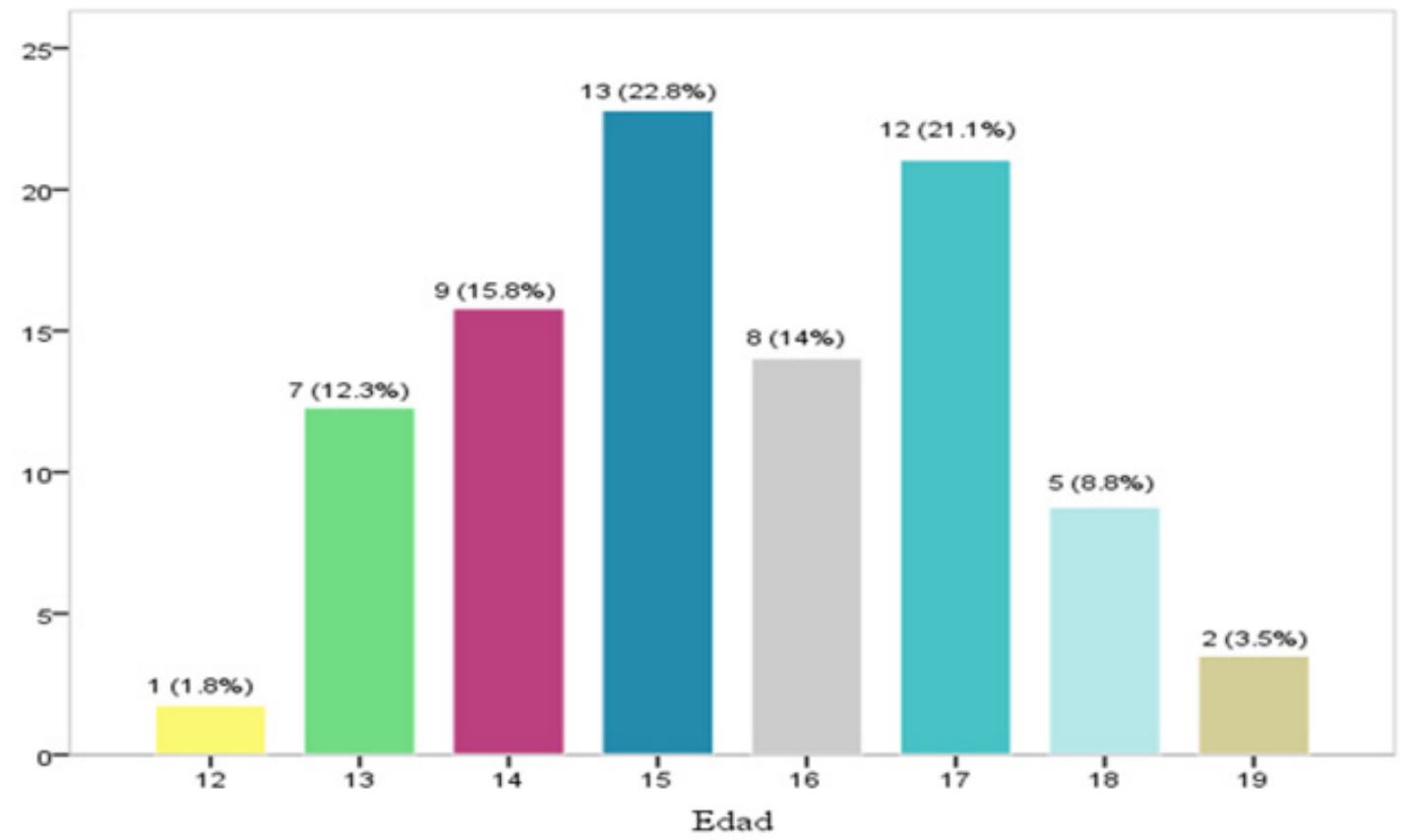

Figura 2.

Edad de inicio de vida sexual en adolescentes

En la tabla $\mathrm{N}^{\circ} 4$ se muestra una variación moderada en cuanto al uso $(64.4 \%)$ y no uso $(38.6 \%)$ de preservativo en la primera relación sexual, de igual manera en el uso $(52.6 \%)$ y no uso $(47.4 \%)$ de preservativo en la última relación sexual. Por otro lado, el $73.7 \%$ no han practicado relaciones sexuales bajo el efecto del alcohol o sustancias psicoactivas, solo el $28.1 \%$ de la población han tenido relaciones sexuales con personas desconocidas, de los cuales el $37.5 \%$ no usaron protección, y el $31.3 \%$ estuvo bajo el efecto de sustancias psicoactivas; finalmente, el $1.8 \%$ indicó haber practicado relaciones sexuales en grupo y con trabajadoras sexuales. 
Tabla 4

Conductas sexuales de riesgo en adolescentes

\begin{tabular}{|c|c|c|c|c|}
\hline & \multicolumn{2}{|l|}{ Sí } & \multicolumn{2}{|l|}{ No } \\
\hline & $\mathbf{n}$ & $\%$ & $\mathbf{n}$ & $\%$ \\
\hline Uso de preservativo en la primera relación sexual & 35 & $61.4 \%$ & 22 & $38.6 \%$ \\
\hline Uso de preservativo en la última relación sexual & 30 & $52.6 \%$ & 27 & $47.4 \%$ \\
\hline $\begin{array}{l}\text { Relaciones sexuales bajo el efecto de alcohol o sustancias } \\
\text { psicoactivas }\end{array}$ & 15 & $26.3 \%$ & 42 & $73.7 \%$ \\
\hline $\begin{array}{l}\text { Relaciones sexuales con personas recién conocidas (en fies- } \\
\text { tas, discotecas, chat, etc.) }\end{array}$ & 16 & $28.1 \%$ & 41 & $71.9 \%$ \\
\hline $\begin{array}{l}\text { Uso de protección en relaciones sexuales con personas re- } \\
\text { cién conocidas }\end{array}$ & 10 & $62.5 \%$ & 6 & $37.5 \%$ \\
\hline $\begin{array}{l}\text { Uso de sustancias psicoactivas en relaciones sexuales con } \\
\text { personas recién conocidas }\end{array}$ & 5 & $31.3 \%$ & 11 & $68.8 \%$ \\
\hline Relaciones sexuales en grupo & 1 & $1.8 \%$ & 56 & $98.2 \%$ \\
\hline Relaciones sexuales con trabajadoras sexuales & 1 & $1.8 \%$ & 56 & $98.2 \%$ \\
\hline
\end{tabular}

En la tabla $\mathrm{N}^{\circ} 5$ se percibe que, en toda la vida sexual, la mayoría de adolescentes ha tenido solo una pareja sexual (59.6\%), en tanto que el $10.5 \%$ y $8.8 \%$ indican que el número de parejas sexuales asciende a tres y dos respectivamente. Por otro lado, el $5.3 \%$ han tenido cuatro y seis parejas sexuales en su vida, mientras que sólo el $1.8 \%$ ha tenido ocho, nueve y catorce parejas sexuales. Respecto a los últimos seis meses de vida sexual, el $83.9 \%$ indica haber tenido una pareja sexual, el $12.9 \%$ dos parejas sexuales y, por último, el 3.2\% presenta catorce parejas sexuales.

Tabla 5

Número de parejas sexuales en adolescentes

\begin{tabular}{lll} 
En toda su vida & $\mathrm{n}$ & $\%$ \\
Una pareja sexual & 34 & 59.6 \\
Dos parejas sexuales & 5 & 8.8 \\
Tres parejas sexuales & 6 & 10.5 \\
Cuatro parejas sexuales & 3 & 5.3 \\
Cinco parejas sexuales & 1 & 1.8 \\
Seis parejas sexuales & 3 & 5.3 \\
Siete parejas sexuales & 2 & 3.5 \\
Ocho parejas sexuales & 1 & 1.8 \\
Nueve parejas sexuales & 1 & 1.8 \\
Catorce parejas sexuales & 1 & 1.8 \\
En los últimos seis meses & & \\
Una pareja sexual & 26 & 83.9 \\
Dos parejas sexuales & 4 & 12.9 \\
Catorce parejas sexuales & 1 & 3.2 \\
\hline
\end{tabular}

En la tabla $N^{\circ} 6$ se aprecia que el $45.6 \%$ usan "a veces" el preservativo en el acto sexual, el $42.1 \%$ "siempre" usan el preservativo, mientras que el $12.3 \%$ lo usan "nunca". Respecto al uso de preservativo, los que dicen "nunca" y "a veces" presentan conductas sexuales de riesgo, lo que se ve representado en un porcentaje de la población encuestada. 
Tabla 6

Frecuencia del uso del preservativo en las relaciones sexuales

$\begin{array}{lll} & \text { n } & \% \\ \text { Nunca } & 7 & 12.3 \% \\ \text { A veces } & 26 & 45.6 \% \\ \text { Siempre } & 24 & 42.1 \%\end{array}$

En la tabla $\mathrm{N}^{\circ} 7$ se observa que la mayor parte de la muestra solo practican sexo vaginal $(10.4 \%)$, el $8 \%$ practica sexo vaginal y oral, el $7 \%$ presenta los tres tipos, y solo el $1 \%$ practica sexo vía vaginal y anal, solo oral y, oral y anal.

Tabla 7

Tipos de relaciones sexuales que practican los adolescentes

\begin{tabular}{lll} 
& $\mathrm{n}$ & $\%$ \\
En conjunto & & \\
Solo vaginal & 21 & $10.4 \%$ \\
Vaginal y oral & 16 & $8 \%$ \\
Vaginal, oral y anal & 14 & $7 \%$ \\
Vaginal y anal & 2 & $1 \%$ \\
Solo oral & 2 & $1 \%$ \\
Oral y anal & 2 & $1 \%$ \\
\hline
\end{tabular}

\section{Prueba de chi-cuadrado}

Respecto a la asociación entre funcionamiento familiar y conductas sexuales de riesgo en adolescentes, la tabla $\mathrm{N}^{\circ} 8$ presenta la correlación existente entre la variable 1 y los ítems de la variable 2. Se evidencia que el estadístico chicuadrado de Pearson toma un valor de 2.398 en el inicio temprano de la vida sexual (.494), 4.107 y 4.579 en el uso de preservativo en la primera (.250) y última (.205) relación sexual respectivamente, y un valor de 1.407 en el caso de relaciones sexuales bajo el efecto de alguna sustancia psicoactiva (.704); así también, se percibe un valor de .887 en cuanto a las relaciones sexuales con personas recién conocidas (.829), siendo los valores de 4.053 y 4.829 en el uso de protección (.256) y haber estado bajo los efectos de algún estimulante psicoactivo (.185) respectivamente, en dichas relaciones sexuales con desconocidos; y por último se muestra un valor de 2.850 en las relaciones sexuales con trabajadoras sexuales (.415); según lo descrito, respecto a las conductas sexuales de riesgo que han sido mencionadas, no se evidencia asociación significativa con la variable funcionamiento familiar dado que la muestra presenta en su mayoría una dinámica familiar entre moderadamente funcional y funcional, y basándonos en la literatura presentada y al análisis teórico personal se deduce que los adolescentes con un funcionamiento familiar adecuado tienden a manifestar minoritariamente conductas sexuales de riesgo; y en los resultados se aprecia una dinámica familiar funcional y bajas conductas sexuales de riesgo, es decir, a mayor funcionalidad familiar (prevalencia de factores protectores como el entorno familiar, elementos personales y psicológicos) menor incidencia de conductas sexuales de riesgo. Asimismo, existe la probabilidad de que tal resultado este influenciado p.e. por el tipo de educación: nacional o particular, debido al "conservadurismo" que algunas instituciones presentan, o también podría asociarse a la cantidad de la muestra descrita en la metodología.

Sin embargo, pese a que la mayoría de conductas sexuales no mostró relación significativa con la variable 1 y luego de traducir dicho resultado en el apartado anterior, se aprecia una excepción con respecto a la conducta sexual "relaciones sexuales en grupo" ya que toma un valor de 13.487 según el estadístico chi-cuadrado de Pearson, con una probabilidad asociada de .004 
mostrando relación significativa con la variable funcionamiento familiar $(\mathrm{p}<.05)$. Tal resultado se fundamenta en que determinado porcentaje de la muestra evaluada práctica relaciones sexuales en grupo que puede deberse a distintos factores predictores del funcionamiento familiar como los determinantes sociales, los estilos de vida durante la adolescencia, el sexo, el grupo de edad, el estatus socioeconómico, y la etnia. Entonces, es más probable la presencia de tal conducta sexual.

Tabla 8

Prueba de chi-cuadrado para conductas sexuales de riesgo en adolescentes

\begin{tabular}{|c|c|c|c|}
\hline Variables & Valor & gl & $\mathbf{P}$ \\
\hline Inicio temprano de vida sexual & 2.398 & 3 & ,494 \\
\hline Uso de preservativo en la primera relación sexual & 4.107 & 3 & ,250 \\
\hline Uso de preservativo en la última relación sexual & 4.579 & 3 & ,205 \\
\hline Relaciones sexuales bajo el efecto de alcohol o sustancias psicoactivas & 1.407 & 3 & ,704 \\
\hline $\begin{array}{l}\text { Relaciones sexuales con personas recién conocidas (en fiestas, discote- } \\
\text { cas, chat, etc.) }\end{array}$ & .887 & 3 & ,829 \\
\hline $\begin{array}{l}\text { Uso de protección en relaciones sexuales con personas recién conoci- } \\
\text { das }\end{array}$ & 4.053 & 3 & ,256 \\
\hline $\begin{array}{l}\text { Uso de sustancias psicoactivas en relaciones sexuales con personas } \\
\text { recién conocidas }\end{array}$ & 4.829 & 3 & ,185 \\
\hline Relaciones sexuales en grupo & 13.487 & 3 & ,004 \\
\hline Relaciones sexuales con trabajadoras sexuales & 2.850 & 3 & ,415 \\
\hline
\end{tabular}

\section{DISCUSIÓN}

En cuanto a la relación entre funcionamiento familiar y relaciones sexuales en grupo, que sería una de las conductas sexuales de riesgo, se evidencia una significancia alta ( $x 2$ $=, 004^{* *} ; p<.05$ ), lo cual puede corresponder a distintos factores predictores del funcionamiento familiar presente en los adolescentes, como los determinantes sociales, los estilos de vida durante la adolescencia, el sexo, el grupo de edad, el estatus socioeconómico, y la etnia (Lima et al., 2012); de esta manera se corrobora lo planteado por Mejía (2019) quien demuestra que a mayor funcionamiento familiar menos probable es la práctica de conductas sexuales de riesgo.

Estas similitudes indican la asociación de las variables ya que un mejor funcionamiento familiar será un factor predictor de bajas conductas de riesgo sexual, demostrando una relación en sentido inverso, lo cual se evidencia en el presente estudio dado que el mayor porcentaje de la población se encuentra en un nivel familiar adecuado y sólo un menor porcentaje de los adolescentes que han iniciado su vida sexual $(28.4 \%)$ muestran conductas sexuales de riesgo, lo cual está asociado a lo mencionado por Flores (2014) quien en su estudio concluyó que, ante una funcionalidad familiar adecuada donde se fomente la comunicación, buena cohesión, clima de confianza óptimo, relaciones interpersonales saludables sin censura de sentimientos, pensamientos o conflictos, existe menor probabilidad de que los adolescentes cometan conductas sexuales de riesgo o cualquier tipo de comportamiento que dañe la salud o integridad de la persona.

Similar a lo mencionado por Narváez et al. (2017) en un estudio realizado en Ecuador demostrando que los adolescentes que provienen de familias disfuncionales donde predomina la comunicación deficiente, patrones comportamentales de agresividad o pasividad tienen más tendencia a incursionar en conductas sexuales de riesgo a edades más tempranas.

En la muestra se evidencia que no hay relación directa entre funcionalidad familiar $y$ las conductas sexuales de riesgo, lo cual conlleva al autoanálisis de la investigación, hallándose distintas limitación que podría estar influenciando en los resultados, y es que en la literatura descrita en el apartado de la introducción se 
muestra a una variedad de autores manifestando la correlación entre ambas variables de estudio en sus investigaciones científicas, empero no se logra apreciar lo mismo en el presente estudio; no obstante, observando y reanalizando los resultados se concluye que la falta de asociación se debería a la dinámica familiar dado que la población encuestada $(n=201)$ en su mayoría presenta un funcionamiento familiar "moderado" (36.8\%) y "adecuado (34.3\%)" percibiéndose en la muestra seleccionada (aquellos que se iniciaron sexualmente $n=57$ ) un porcentaje mínimo de conductas de riesgo sexual, en tanto que la población restante $(n=144)$ mencionó no haber iniciado su vida sexual. Por tanto, las conductas sexuales de riesgo que no estuvieron asociadas a la variable funcionamiento familiar tales como: uso de preservativo en la primera y última relación sexual, relaciones sexuales bajo el efecto de alcohol o sustancias psicoactivas, relaciones sexuales con personas recién conocidas, uso de protección y sustancias psicoactivas en relaciones sexuales con personas recién conocidas y sexo con trabajadoras sexuales; demuestran una relación inversa entre ambas variables, es decir, hay un adecuado funcionamiento familiar por lo que la incidencia en conductas sexuales de riesgo es mínima.

Tal resultado se asocia al estudio realizado por Lavielle et al. (2014) en México quienes concluyen que a mayor deficiencia de atributos familiares mayor será la predisposición a conductas de riesgo sexual en los adolescentes; y al estudio realizo por Pérez et al., (2018) en Ecuador quienes refieren que a menos funcionalidad familiar mayor incidencia de conductas de riesgo.

En síntesis, una familia funcional fomenta las buenas relaciones intrafamiliares, prioriza el desarrollo de una buena cohesión y clima de confianza donde padres e hijos interactúan sin la necesidad de reprimir pensamientos, sentimientos o conflictos; contrariamente a las familias disfuncionales donde la desintegración, la falta de valores y el diálogo pasivo-agresivo convierte al adolescente en un ser vulnerable que fácilmente irrumpa en conductas que ponen en riesgo su salud física, emocional y moral, tal como es la problemática del presente estudio. En tal sentido, se observa que a mayor funcionalidad familiar menor probabilidad de que el adolescente presente conductas sexuales de riesgo.

\section{Declaración de financiamiento y de conflictos de interés:}

El estudio fue financiado por los autores, quienes declaran no tener conflictos de interés

\section{Correspondencia \\ Analy Quiroz Muñoz}

Correo electrónico:

analyquiroz@upeu.edu.pe

\author{
Jessy Milagros Johana Estelo Quispe \\ Correo electrónico: \\ jessyestelo@upeu.edu.pe
}

\section{REFERENCIAS}

Águila, G., Díaz, J., Díaz, P. (2018). Adolescencia temprana y parentalidad: Fundamentos teóricos y metodológicos acerca de esta etapa y su manejo. Medisur, 15(5), 694-700. http://scielo. sld.cu/scielo.php?script=sci_arttext\&pid=S1727897X2017000500015

Alarcón, R. (1998). La teoría clásica de los Test: Logros y limitaciones. En: Nuevos modelos en la medición psicológica (pp. 9-30). Lima: USMP.

Albornoz, P., Encalada, Z., \& Meza, K. (2018). Funcionamiento familiar percibido y conuctas de riesgo en adolescentes del Colegio Nacional Cesar Vallejo, Amarilis-Paucarbamba, 2018. Universidad Nacional Hermilio Valdizán. http://repositorio.unheval.edu.pe/bitstream/ handle/UNHEVAL/4228/TEN01120A36. pdf? sequence $=1$ \&isAllowed $=y$

Aliaga, L. (2017). Nivel de conocimiento sobre las infecciones de transmisión sexual $y$ conductas sexuales de riesgo en escolares mujeres de una institución educativa de Lima, 2016. Universidad Nacional Mayor de San Marcos. http://cybertesis.unmsm.edu. pe/bitstream/handle/cybertesis/5851/Aliaga_ el. pdf? sequence=1\&isAllowed=y

Andrade, P., Betancourt, D., \& Palacios, J. (2006). Factores familiares asociados a la conducta sexual en adolescentes. Revista Colombiana de Psicología, 1(15), 91-101. https://www. researchgate.net/publication/26848804_ Factores_familiares_asociados_a_la_conducta_sexual_en_adolescentes

Apaza, B., Quiroz, A., \& Estelo, JM. (2017). Estilos de afrontamiento y conductas sexuales de riesgo en adolescentes de tres instituciones educativas del distrito de Lurigancho. Universidad Peruana 
Unión, Facultad de Ciencias de la Salud 1(1), 1-72.

Breinbauer, C. y Maddaleno, M. (2005). Youth: Choices and change. Promoting healthy behaviors in adolescents. Scientific and Technical Publication, 1(594), 1-1025. https://books. google.com.co/books?id=goqyfO8KkFIC\&print sec=frontcover \&dq=Youth:+Choices+and+cha nge.+Promoting+healthy+behaviors+in+adole scents. \&hl=es $-419 \&$ sa $=X \&$ ved $=0$ ahUKEwiMs$7 \mathrm{q} \times 77 \times$ a A h U G J t $8 \mathrm{~K} \mathrm{H} \mathrm{T}$ dC7wQ6AEIKjAA\#v=onepage\&q=Youth $\% 3 A \% 2 \overline{0}$ $\mathrm{C}$ h o i c e s $\% 20$ and $\% 20 \mathrm{ch}$ an g e. $\% 20$ Promoting $\% 20$ healthy $\% 20$ behaviors $\% 20$ in $\% 20$ adolescents. \&f=false

Casasa, P., Ramírez, E., \& Guillén, R. (2016). Salud familiar y enfermería. México El Manual Moderno. https://books.google. com.pe/books? id=-zPVDAAAQBAJ\&prin tsec $=$ frontcover $\& \mathrm{hl}=\mathrm{es} \&$ source=gbs_ge summary_r\&cad $=0 \# v=$ onepage $\& q \& f=$ false

Castellón, S. y Ledesma, A. (2012). El funcionamiento familiar y su relación con la socialización infantil. Proyecciones para su estudio en una comunidad suburbana de Sancti Spíritus. Cuba". Contribuciones a las ciencias sociales, 7. http:// www.eumed.net/rev/cccss/21/ccla.html

Espada, J., Quiles, M., \& Méndez, F. (2003). Conductas sexuales de riesgo y prevención del SIDA en la adolescencia. Papeles Del Psicólogo, 24(85), 29-36. http://www.redalyc.org/articulo. oa?id=77808504

Fierros, L. E., Rivera, B. M., \& Piña, J. A. (2011). Sexual Behavior With Casual Partners Among University Women. Revista Iberoamericana de Psicología Y Salud, 2(2), 165-183. http://www. redalyc.org/resumen.oa?id=245118507003

Flores, L. (2014). Funcionamiento familiar y su relación con las conductas de riesgo en adolescentes del nivel secundario de la Institución Educativa Enrique Paillardelle Tacna - 2013. Universidad Nacional Jorge Basadre Grohmann de Tacna. http://repositorio.unjbg. edu.pe/bitstream/handle/UNJBG/328/TG0178. pdf?seque nce=1\&isAllowed=y

González, E., Molina, T., Montero, A., Martínez, V., \& Leyton, C. (2007). Comportamientos sexuales y diferencias de género en adolescentes usuarios de un sistema público de salud universitario. Revista Médica de Chile, 135, 1261-1269. http:// doi.org/10.4067/S0034-98872007001000005

Gutgesell, M. y Payne, N. (2004). Issues of adolescent psychological development in the 21st century. Pediatrics in Review, 25 (3), 79-85. doi: 10.1542/ pir.25-3-79
Hernández, R., Fernández, C., \& Baptista, P., (2014). Metodología de la Investigación. Editorial McGraw Hill.

Instituto Nacional de Estadísitica e Informática INEI. (2015). Perú: Encuesta Demográfica y de Salud Familiar. Instituto Nacional de Estadística e Informática. https://www.inei.gob.pe/media/ MenuRecursivo/publicaciones_digitales/Est/ Lib1211/pdf/Libro.pdf

Korenromp, E., Rowley, J., Alonso, M., Mello, M., Wijesooriya, S., Mahiané, G., ... Taylor, M. (2019). Global burden of maternal and congenital syphilis and associated adverse birth outcomes: estimates for 2016 and progress since 2012. PLOS ONE, 14(2), e0211720. http://doi.org/10.1371/journal. pone.0211720

Lavielle, P., Jiménez, F., Vásquez, A., Aguirre, M., Castillo, M., \& Vega, S. (2014). Impacto de la familia en las conductas sexuales de riesgo de los adolescentes. Revista Médica Del Instituto Mexicano Del Seguro Social, 52(1), 38-43. https://www.medigraphic.com/pdfs/imss/im-2014/ im141j.pdf

Lima, M., Neves, S., \& Lima, J. (2012). Actitudes de adolescentes hacia la salud: evaluación de un programa escolar de promoción de la salud en Sevilla, España. Salud Colectiva, 8(1), 4760. https://www.scielosp.org/article/scol/2012. v8n1/47-60/

Llaza, R., \& Ocoruro, F. (2018). Funcionamiento familiar y conductas sexuales de riesgoo en adolescentes. Universidad Nacional de San Agustín de Arequipa. http://repositorio.unsa. edu.pe/bitstream/handle/UNSA/7345/PSIlayri. pdf? sequence=1\&isAllowed=y

Looker, K., Magaret, A., Turner, K., Vickerman, P., Gottlieb, S., \& Newman, L. (2015). Global estimates of prevalent and incident herpes simplex virus type 2 infections in 2012. PLOS ONE, 10(1), e114989. http://doi.org/10.1371/ journal.pone.0114989

López, PL. (2004). Población Muestra y Muestreo. Punto Cero, 09(08), 69-74. Recuperado por http://www.scielo.org.bo/scielo.php?script=sci arttext\&pid=S1815-02762004000100012\&lng=es \&tIng=es.

Losa, R., \& Gonzáles, M. (2018). Conductas sexuales en adolescentes de una escuela secundaria en Mérida, Yucatán, México. Revista Biomédica, 29(3), 81-87. http://doi.org/10.32776/revbiomed. v29i3.625

Louro, I. (2003). La familia en la determinación de la salud. Revista Cubana Salud Pública, 29(1), 4851. http://scielo.sld.cu/pdf/rcsp/v29n1/spu07103. pdf 
Louro, I. (2004). Modelo teórico - metodológico para la evaluación de salud del grupo familiar en la atención primaria. Escuela Nacional de Salud Pública. http://www.sld.cu/galerias/pdf/sitios/ revsalud/tesis_isabel_louro.pdf

Mejía, N. (2019). Funcionamiento familiar $y$ conductas de riesgo sexual en estudiantes universitarios de una Institución Pública de Lima. Universidad Nacional Federico Villareal. http://repositorio.unfv.edu.pe/bitstream/handle/ UNFV/2815/UNFV MEJIA VIDAL NERI AMANDA_SEGUNDĀ_ESPEC $\bar{C} I A L I D A \bar{D} \_2019$. pdf? sequence $=1 \&$ isAllowed $=y$

Ministerio de Salud - MINSA. (2017). Análisis de la Situación Epidemiológica del VIH/SIDA en el Perú (1983-2017). In Centro Nacional de Epidemiología, Prevención y Control de Enfermedades (pp. 1-6). http://www.dge.gob.pe/ portal/docs/vigilancia/sala/2017/SE23/vih.pdf

Mola, R., Araújo, R., Oliveira, J., Cunha, S., Souza, G., Ribeiro, L., \& Pitangui, A. (2017). Association between the number of sexual partners and alcohol consumption among schoolchildren. Journal de Pediatria, 93(2), 192-199. http://doi. org/10.1016/j.jped.2016.05.003

Narváez, G., Pabón, K., León, V., Guzmán, C., \& Tapia, I. (2017). Estudio exploratorio de la incidencia de los hogares disfuncionales en la iniciación sexual temprana de los adolescentes. Ecos de La Academia, 6(5), 169-179. https://www.researchgate. net/publication/322722005_ESTUDIO_ EXPLORATORIO_DE_LA_INCIDENCIA_DE_LOS_HOGARES_DISFUNCIONALES_EN_LA_ INICIACION_SEXUAL_TEMPRANA_DE_LOS_ ADOLESCEN̄TES/citations

OMS. (2012). Prevenir el embarazo precoz y los resultados reproductivos adversos en adolescentes en los países en desarrollo: las evidencias. Departmento de salud materna, del recién nacido, del niño y el adolescente. https:// apps.who.int/iris/bitstream/handle/10665/78253/ WHO_FWC_MCA_12_02_spa.pdf?sequence=1

OMS. (2016). Estrategia mundial del sector de la salud contra las infecciones de trasmisión sexual 20162021: hacia el fin de las ITS. Organización Mundial de la Salud. https://apps.who.int/iris/bitstream/ handle/10665/250253/WHO-RHR-16.09-spa. pdf;jsessionid=30C578FF879EC69153 870D7BA 1F5DBE5? sequence $=1$

OMS. (2018). Report on global sexually transmitted infection surveillance. Organización Mundial de la Salud. https://apps.who.int/iris/bitstream/hand le/10665/277258/9789241565691-eng.pdf?ua=1
Panuera, M. (2018). Funcionalidad familiar, estilos parentales y sintomatología depresiva en adolescentes de tercero, cuarto y quinto año de secundaria de una institución educativa estatal en Chorrillos. Universidad Nacional Mayor de San Marcos. http://cybertesis.unmsm.edu.pe/ bitstream/handle/cybertesis/10133/Delgado_ va. pdf?sequence $=1 \&$ isAllowed $=y$

Pérez, Y., Apupalo, M., \& Creagh, I. (2018). Funcionamiento familiar y conducta sexual de riesgo en adolescentes de la comunidad de Yanayacu, 2015-2016. Revista Habanera de Ciencias Médicas, 17(5), 789-799. http:// scielo.sld.cu/pdf/rhcm/v17n5/1729-519Xrhcm-17-05-789.pdf

Restrepo, O. (2010). Crecieminto y desarrollo en el adolescente. Universidad de Anioquía, 1(3), 68-79. https://www.researchgate.net/ publication/301890766_Crecimiento_y_ desarrollo_en_el_adolescente

Sanjosé, S., Diaz, M., Castellsagué, X., Clifford, G., Bruni, L., Muñoz, N., \& Bosch, X. (2007). Worldwide prevalence and genotype distribution of cervical human papillomavirus DNA in women with normal cytology: a meta-analysis. The Lancet Infectious Diseases, 7(7), 453-459. http:// doi.org/10.1016/S1473-3099(07)70158-5

Tarazona, D. (2005). El comportamiento sexual y su vinculación con variables psicológicas y demográficas en estudiantes de quinto año de eduación secundaria. Universidad Mayor de San Marcos, 1(1), 1-148.

Recibido: $11 / 07 / 2021$

Aceptado: 25/10/2021 